\title{
Superconductivity in single-crystalline aluminum- and gallium-hyperdoped germanium
}

\author{
Slawomir Prucnal,,${ }^{1, *}$ Viton Heera, ${ }^{1}$ René Hübner, ${ }^{1}$ Mao Wang, ${ }^{1}$ Grzegorz P. Mazur, ${ }^{2,3}$ Michał J. Grzybowski, ${ }^{2,3}$ Xin Qin, ${ }^{4}$ \\ Ye Yuan, ${ }^{1,5}$ Matthias Voelskow, ${ }^{1}$ Wolfgang Skorupa, ${ }^{1}$ Lars Rebohle, ${ }^{1}$ Manfred Helm, ${ }^{1}$ Maciej Sawicki, ${ }^{2}$ and Shengqiang Zhou ${ }^{1}$ \\ ${ }^{1}$ Helmholtz-Zentrum Dresden-Rossendorf, Institute of Ion Beam Physics and Materials Research, \\ Bautzner Landstraße 400, D-01328 Dresden, Germany \\ ${ }^{2}$ Institute of Physics, Polish Academy of Sciences, Aleja Lotnikow 32/46, PL-02668 Warsaw, Poland \\ ${ }^{3}$ International Research Center MagTop, Institute of Physics, Polish Academy of Sciences, Aleja Lotnikow 32/46, PL-02668 Warsaw, Poland \\ ${ }^{4}$ Hefei National Laboratory for Physical Sciences at the Microscale, University of Science and Technology of China, \\ Hefei, Anhui 230026, China \\ ${ }^{5}$ Physical Science and Engineering Division, King Abdullah University of Science and Technology, 23955-6900 Thuwal, Saudi Arabia
}

(Received 18 December 2018; revised manuscript received 15 March 2019; published 9 May 2019)

\begin{abstract}
Superconductivity in group IV semiconductors is desired for hybrid devices combining both semiconducting and superconducting properties. Following boron-doped diamond and $\mathrm{Si}$, superconductivity has been observed in gallium-doped Ge; however, the obtained specimen is in polycrystalline form [Phys. Rev. Lett. 102, 217003 (2009)]. Here we present superconducting single-crystalline Ge hyperdoped with gallium or aluminum by ion implantation and rear-side flash lamp annealing. The maximum concentration of $\mathrm{Al}$ and Ga incorporated into substitutional positions in $\mathrm{Ge}$ is 8 times higher than the equilibrium solid solubility. This corresponds to a hole concentration above $10^{21} \mathrm{~cm}^{-3}$. Using density functional theory in the local-density approximation and pseudopotential plane-wave approach, we show that the superconductivity in $p$-type Ge is phonon mediated. According to the $a b$ initio calculations, the critical superconducting temperature for Al- and Ga-doped Ge is in the range of $0.45 \mathrm{~K}$ for 6.25 at.\% of dopant concentration, being in qualitative agreement with experimentally obtained values.
\end{abstract}

DOI: 10.1103/PhysRevMaterials.3.054802

\section{INTRODUCTION}

Since the discovery of superconductivity in diamond [1] with a boron content above the equilibrium solid solubility (ESS), many studies have been performed to find new "superconducting semiconductors." Such a materials class would enable the monolithic integration of quantum and conventional electronics [2]. Indeed, several groups found superconductivity, even in the technologically more relevant semiconductors such as $\mathrm{Si}$ [3], Ge [4], or SiC [5], after a heavy hole doping. A brief introduction into the research field of superconducting semiconductors was given in recent review articles [6-9].

The term "superconducting semiconductor" is a bit misleading, since in a semiconductor, the carriers necessary for the Cooper pair condensate freeze out at low temperatures, and superconductivity is impossible. Therefore, the semiconductor has to be heavily doped above the metal-insulator transition (MIT). It turned out that an acceptor concentration in excess of 1 at.\% (i.e., above $5 \times 10^{20} \mathrm{~cm}^{-3}$ ) is required to induce the superconductivity in germanium. Such concentration is higher than the ESS of typical acceptors in Ge. Hyperdoping, however, is difficult to achieve and requires nonequilibrium doping techniques, such as a high-pressure high-temperature synthesis [1] or chemical vapor deposition [8] in the case of diamond, gas immersion laser doping $[3,10,11]$, and high-fluence ion implantation combined with

\footnotetext{
*Corresponding author: s.prucnal@hzdr.de
}

rapid thermal annealing (RTA) or flash lamp annealing (FLA) [4,12-15] for Si and Ge. Among these doping schemes, ion implantation followed by FLA is best adopted to the current semiconductor technology.

Despite advanced nonequilibrium doping techniques, hyperdoped semiconductors are in most cases inhomogeneous materials with dopant concentration fluctuations [16] up to cluster or nanoprecipitate formation [13]. Moreover, dopant segregation at grain boundaries in polycrystalline materials or at interfaces to technologically relevant capping layers is a next serious problem [17]. There is experimental evidence that in some semiconductor-acceptor systems, $\mathrm{Si}: \mathrm{Ga}$, for example, amorphous acceptor-rich nanoprecipitates $\left(c_{\mathrm{Ga}}>20\right.$ at.\%) are vital for superconductivity [13]. Granularities of the superconducting condensates have been also obtained in boron-doped diamond $[18,19]$. Such granular superconductors can be modeled by a random network of Josephson junctions and exhibit a superconductor-insulator transition [20], as observed, e.g., in Si:Ga [21,22]. The presence of the superconductor-insulator transition clearly reveals the inhomogeneous character of the superconductor. Due to local superconducting regions, even in the insulating state such hyperdoped semiconductors demonstrate nonlinear transport phenomena [22] and anomalous large magnetoresistance [23].

However, for a perfect monolithic integration of superconducting nanocircuits in semiconductor devices, a homogeneous and single-crystalline structure is desirable. It remains an unresolved question whether superconducting semiconductor films of sufficient quality can be fabricated at all 
by today's top-down selective doping technologies and which semiconductor-acceptor combination is most promising. Since the tendency for disorder and cluster formation by hyperdoping increases with the covalent bond strength of the semiconductor and decreases with growing acceptor solid solubility, the Ge:Ga system appears to be favorable compared to diamond:B and Si:B [14]. Previous studies demonstrated that conventional implantation doping of $\mathrm{Ge}$ with $\mathrm{Ga}$ enables a maximum hole concentration of $6.6 \times 10^{20} \mathrm{~cm}^{-3}$ after annealing at $450^{\circ} \mathrm{C}$ for $1 \mathrm{~h}$ [24]. Higher temperatures of conventional long-term annealing led to Ga clustering. In order to reduce acceptor diffusion and clustering, FLA in the millisecond range without layer melting is an appropriate method [25-28]. With this method hole concentrations up to $1.4 \times 10^{21} \mathrm{~cm}^{-3}$ and superconductivity at critical temperatures below $0.5[4,15]$ and $2.0 \mathrm{~K}$ [29] have been achieved in Ge layers with about 6 and 8 at.\% $\mathrm{Ga}$ content, respectively. Unfortunately, the layers are nanocrystalline $[4,15]$, and the activation level of the Ga acceptors varies from sample to sample up to a factor of 2, which is due to the formation of Ga-rich nanoprecipitates [29]. Single-crystalline Ge:Ga has been obtained by RTA [14]. However, in this case, a large amount of the $\mathrm{Ga}$ atoms accumulates as an amorphous film at the $\mathrm{SiO}_{2} / \mathrm{Ge}$ interface. This interface layer becomes superconducting below $6 \mathrm{~K}$, which is similar to the critical temperature of Ga clusters.

In this paper we show that an optimized FLA process can be used to fabricate single-crystalline, superconducting layers of hyperdoped $p$-type Ge. In addition to Ga doping, we also investigate Al doping. Similar to $\mathrm{Ga}, \mathrm{Al}$ has high ESS but a higher diffusivity than $\mathrm{Ga}$ and is more difficult to activate. As shown recently, ion implantation of $\mathrm{Al}$ into Ge followed by conventional thermal annealing results in a maximum hole concentration of only $1 \times 10^{20} \mathrm{~cm}^{-3}$ [30]. Here we show that the maximum carrier concentration in $\mathrm{Ga}$ - and $\mathrm{Al}$-implanted Ge followed by FLA exceeds $10^{21} \mathrm{~cm}^{-3}$. FLA suppresses the dopant diffusion and segregation. The recrystallized Ge is single crystalline with critical temperatures of $T_{C} \sim 0.5 \mathrm{~K}$. Moreover, first-principles investigation of superconductivity in Al-doped and Ga-doped Ge using ab initio calculations within the Eliashberg-McMillan theory reveals that the $\mathrm{Ga}: \mathrm{Al}$ system behaves similar to a Ge:Ga covalent superconductor, where the critical temperature can be tuned by the carrier concentration.

\section{EXPERIMENTAL}

\section{A. Sample fabrication}

$N$-type (Sb-doped, $\rho>10 \Omega \mathrm{cm}$ ), (100)-oriented Ge wafers are used as substrates for acceptor implantation in order to electrically isolate the processed layer from the substrate by formation of a $p-n$ junction. First, a 30-nm-thick $\mathrm{SiO}_{2}$ cover layer is sputter-deposited to protect the Ge surface during ion implantation and annealing. Then the wafers are implanted with $\mathrm{Ga}$ or $\mathrm{Al}$ ions with different fluencies of 1 , 2 , and $4 \times 10^{16} \mathrm{~cm}^{-2}$ and energies of $100 \mathrm{keV}$ for $\mathrm{Ga}^{+}$and $50 \mathrm{keV}$ for $\mathrm{Al}^{+}$ions. The implantation energies are chosen in such a way that the acceptor profiles are similar, with a maximum acceptor concentration at a depth of $60 \mathrm{~nm}$, as predicted by the SRIM simulation code [31]. Figure 1(a) shows the calculated $\mathrm{Ga}$ and $\mathrm{Al}$ distributions implanted into the $\mathrm{SiO}_{2} / \mathrm{Ge}$ wafers for an ion fluence of $2 \times 10^{16} \mathrm{~cm}^{-2}$. The peak concentration and the depth distribution of $\mathrm{Al}$ and $\mathrm{Ga}$ within Ge are different for the same ion fluence and similar projected ion range $\mathrm{R}_{\mathrm{p}}$. After implantation, a heavily doped amorphous surface layer of about $120 \mathrm{~nm}$ width with a relatively sharp interface to the single-crystalline Ge substrate was formed (see Supplemental Material, Fig. S1a) [15,32]. The presence of a sharp amorphous/crystalline interface is an important precondition for the explosive solid-phase epitaxy process which appears during millisecond-range FLA of the implanted layer [29].

The $\mathrm{Al}$ peak concentration is about 6 at. $\%$, whereas the Ga concentration exceeds 10 at.\%. This is due to different interactions of light ( $\mathrm{Al})$ and heavy (Ga) elements with germanium during the ion-implantation process. This is due to different stopping power and energy loss straggling for different ions within the solid. For heavier ions the stopping power and the energy loss straggling are higher, causing a smaller FWHM of the depth distribution of the implanted ions and, in consequence, a higher peak concentration for the same ion fluence.

In order to activate the dopants and recrystallize the implanted layer, we have used a strongly nonequilibrium thermal processing, i.e., flash lamp annealing. Implanted samples were annealed either from the front side (f-FLA) or from the rear side (r-FLA) with an energy density deposited to the sample surface in the range of $50-130 \mathrm{~J} \mathrm{~cm}^{-2}$. The annealing time was 3,6 , or $20 \mathrm{~ms}$. The influence of the annealing time on the recrystallization process of the implanted layer is presented in Supplemental Material (see Fig. S1b [15,32]). Figure 1(b) shows the temperature distribution within the implanted layer after front- and rear-side FLA for $20 \mathrm{~ms}$. The f-FLA leads to a partial epitaxial regrowth of the implanted layer and to the formation of polycrystalline hyperdoped $\mathrm{Ge}$ at the surface [15]. Taking into account the wavelength spectrum of the Xe lamps in the FLA system (300-800 nm) and the optical properties of $\mathrm{Ge}$, the main part of the flash light is absorbed by implanted Ge within $50 \mathrm{~nm}$ from the surface. This causes a temperature gradient within the implanted layer. For a short moment (in the submicrosecond range), the surface is much hotter than the amorphous/crystalline interface. Also, the threshold energy needed for crystalline seed nucleation is lower than the energy needed for the epitaxial regrowth [33]. Therefore, during f-FLA, the recrystallization of the implanted layer starts from the surface and a polycrystalline layer is formed. In order to avoid the formation of such a polycrystalline layer at the top of implanted $\mathrm{Ge}$, we developed the rear-side FLA process [28]. In this case, the implanted sample is annealed from the rear side and the heat is transferred through the wafer to the implanted surface. Using r-FLA, the amorphous/crystalline interface is heated first. Therefore, before the surface temperature reaches the level needed for crystalline seed nucleation, the whole implanted layer is recrystallized due to the explosive solid-phase epitaxy [28]. We have found that using a $400-\mu \mathrm{m}$-thick Ge layer the optimal annealing time for rear-side annealing is $20 \mathrm{~ms}$. RTA and pulsed laser annealing (PLA) are alternatives to FLA annealing techniques. During RTA, similar to the r-FLA process, 

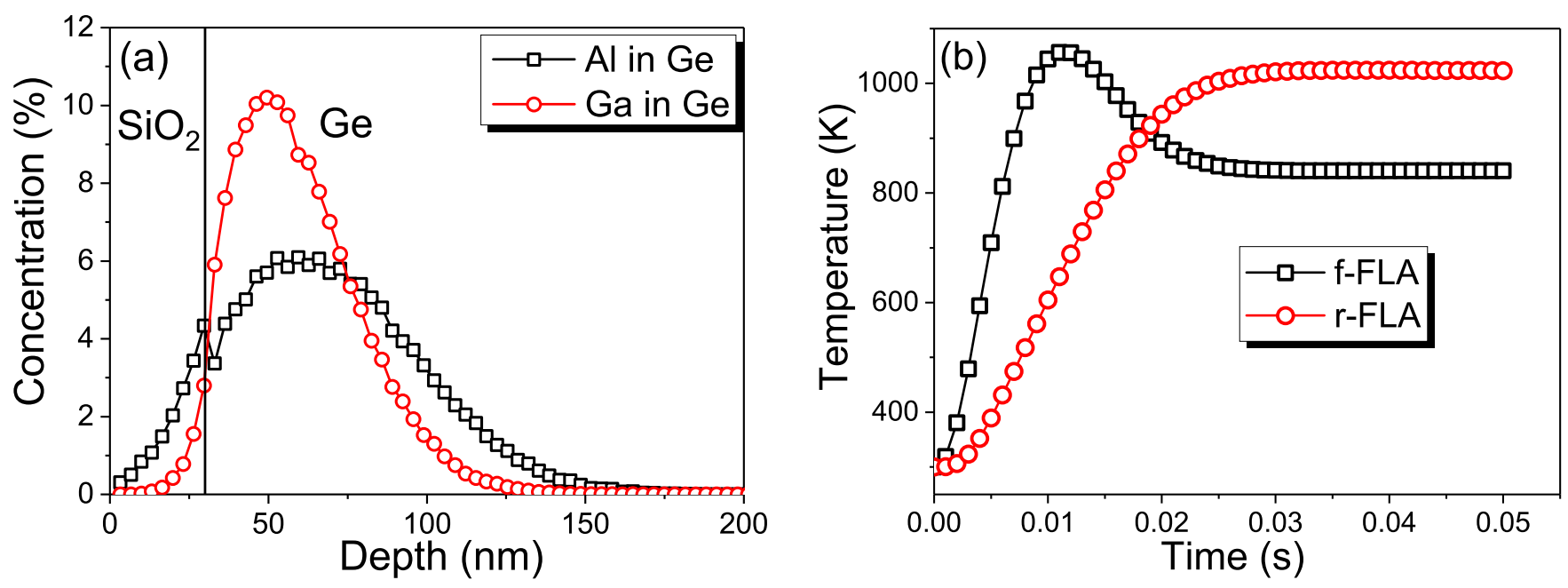

FIG. 1. The Ga and Al depth distribution in Ge obtained by the SRIM code for an ion-implantation fluence of $2 \times 10^{16} \mathrm{~cm}^{-2}$ (a) and simulated temperature distribution at the implanted surface during $20 \mathrm{~ms}$ FLA from the front side (f-FLA, black curve) and from the rear side (r-FLA, red curve) (b).

the implanted layer recrystallizes via solid-phase epitaxy. But due to much smaller heating rate the recrystallization speed of the implanted layer is significantly slower than the diffusion of dopants. As a consequence, the implanted elements with concentration higher than the solid solubility are only partially incorporated into the crystal lattice and form clusters. In contrast to FLA and RTA, during PLA the annealing layer recrystallizes via liquid phase epitaxy. The typical pulse length for PLA is in the nanosecond range, and the total annealing time is in the range of tens of microseconds. The solidification/recrystallization speed observed during PLA is similar to the explosive solid-phase epitaxy after FLA. But the diffusion coefficient of dopants in the liquid phase is a few orders of magnitude higher than in the solids. Hence, during PLA dopants often diffuse towards the surface and form a dopantrich but nonactivated layer. According to our experience, only the millisecond-range annealing provides enough energy to activate explosive solid-phase epitaxy, which is crucial for the formation of single-crystalline hyperdoped germanium.

\section{B. Characterization techniques}

The crystallization process of the Al- and Ga-implanted and annealed samples is studied using Rutherford backscattering-channeling spectrometry (RBS/C). The $\mathrm{RBS} / \mathrm{C}$ measurements are performed on the samples before

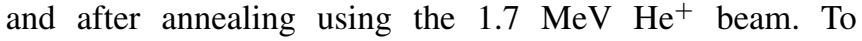
investigate the microstructural properties of the implanted Ge layer, cross-sectional bright-field transmission electron microscopy (TEM) investigations are performed in a Titan 80-300 (FEI) microscope operated at an accelerating voltage of $300 \mathrm{kV}$. High-angle annular dark-field scanning transmission electron microscopy (HAADF-STEM) imaging and spectrum imaging based on energy-dispersive X-ray spectroscopy (EDXS) are performed at $200 \mathrm{kV}$ with a Talos F200X microscope equipped with a Super-X EDXS detector system (FEI). Prior to TEM analysis, the specimen mounted in a high-visibility low-background holder was placed for $10 \mathrm{~s}$ into a model 1020 Plasma Cleaner (Fischione) to remove contaminations. The optical properties are investigated by micro-Raman spectroscopy. The phonon spectra were obtained in backscattering geometry in the range of $100-600 \mathrm{~cm}^{-1}$ using a 532-nm Nd:YAG laser with a liquid-nitrogen-cooled CCD camera.

The concentration of carriers in the implanted and annealed samples was estimated from temperature-dependent Hall-effect measurements in van der Pauw configuration. The thickness of the doped layer was extracted from the RBS data under the assumption that the diffusion of implanted elements during 20-ms pulse annealing can be neglected. The electrical properties of the annealed samples are measured at millikelvin temperatures in a dry dilution refrigerator (Triton 400 by Oxford Instruments), which allows sweeping temperature in the range from $10 \mathrm{mK}$ to $30 \mathrm{~K}$. Four-probe ac measurements were taken using the ac lock-in method with an excitation current of $10 \mathrm{nA}$ and frequency $127 \mathrm{~Hz}$. The existence of superconducting states in hyperdoped $p$-type Ge was predicted by ab initio calculations within the Eliashberg/McMillan theory.

\section{RESULTS AND DISCUSSION}

\section{A. Microstructure}

The recrystallization process of ion-implanted and flashlamp-annealed Ge is investigated using RBS random (RBS/R) and channeling (RBS/C) spectrometry. Since Al and Ga are lighter than $\mathrm{Ge}$, they unfortunately cannot be measured directly by RBS. However, the ratio between the yields of the $\mathrm{RBS} / \mathrm{C}$ and $\mathrm{RBS} / \mathrm{R}$ spectra $\left(\chi_{\min }\right)$ is a measure of the crystalline quality of the sample. In our case, $\chi_{\min }$ for Al- and Ga-hyperdoped Ge after FLA is in the range of $(5 \pm 1) \%$ (see Fig. 5), which is slightly higher than $\chi_{\min }$ for the virgin $\mathrm{Ge}$. Moreover, the RBS/C spectra recorded from the as-implanted samples provide information about the thickness of the amorphized layer, which is needed to calculate the carrier density using Hall-effect measurements.

Figure 2 shows the RBS/R and RBS/C spectra obtained from the Al-doped sample before and after r-FLA, from the Ga-doped sample after r-FLA and from virgin Ge. As 


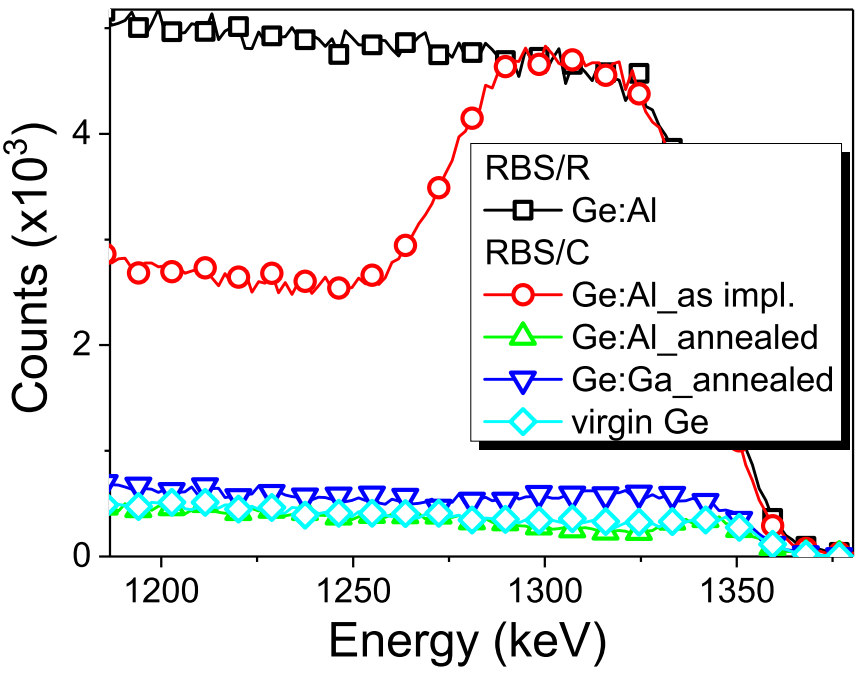

FIG. 2. The RBS/R and RBS/C spectra obtained from Al- and Ga-hyperdoped Ge. The RBS/C spectrum recorded from virgin $\mathrm{Ge}$ is shown as well. The concentrations of $\mathrm{Al}$ and $\mathrm{Ga}$ in $\mathrm{Ge}$ are at the level of $2 \times 10^{21} \mathrm{~cm}^{-3}$.

expected, the RBS/C spectrum obtained from the asimplanted sample reveals the formation about a 120-nm-thick amorphous surface layer. The thickness of the amorphous layer is calculated based on the RBS data using the RUMP Software. After 20-ms r-FLA with an energy density of $120 \mathrm{~J} \mathrm{~cm}^{-2}$, the yield of RBS/C spectrum drops down to the level registered from the virgin Ge wafer. This behavior points to an epitaxial regrowth of the implanted layer during r-FLA. Moreover, we can conclude that $\mathrm{Al}$ atoms are incorporated into the lattice of Ge. Taking into account that the solid solubility of $\mathrm{Al}$ in $\mathrm{Ge}$ is in the range of $5 \times 10^{20} \mathrm{~cm}^{-3}$, the investigated sample contains 4 times more $\mathrm{Al}$ in substitutional positions than the solid solubility limit. Such a gain is only possible due to the strongly nonequilibrium character of the process. The absence of significant dechanneling suggests that the formation of $\mathrm{Al}$ clusters is also suppressed by the millisecond-range r-FLA.

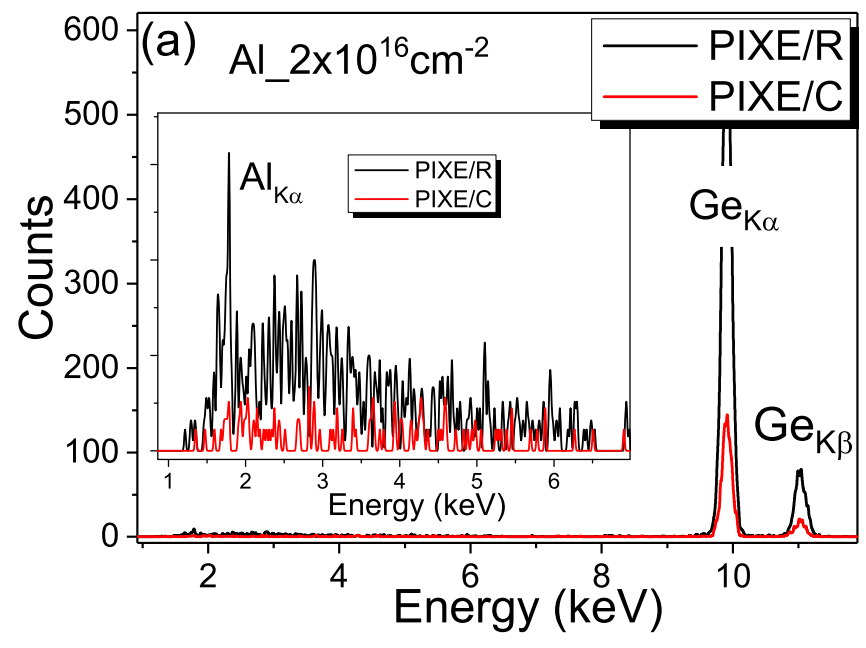

In the case of Ga-hyperdoped Ge after r-FLA, the RBS/C spectrum also reveals full incorporation of $\mathrm{Ga}$ into the $\mathrm{Ge}$ lattice. The yield of the RBS/C spectrum obtained from virgin $\mathrm{Ge}$ and the Ga-doped sample is at the same level, meaning that the Ga-implanted sample behaves the same way as Aldoped Ge after r-FLA. In both cases, the ESS limit has been overcome by 4 times.

In order to clarify the lattice position of $\mathrm{Al}$ and $\mathrm{Ga}$ within $\mathrm{Ge}$, we performed particle-induced $\mathrm{x}$-ray emission (PIXE) spectroscopy in the random and channeling direction. Figures 3(a) and 3(b) show the PIXE spectra obtained from the Al- and Ga-doped samples, respectively. The peaks are identified as the characteristic $\mathrm{x}$-ray emissions of the $\mathrm{Al}_{\mathrm{K} \alpha}(1.78 \mathrm{keV}), \mathrm{Ga}_{\mathrm{K} \alpha}(9.27 \mathrm{keV}), \mathrm{Ge}_{\mathrm{K} \alpha}(9.85 \mathrm{keV})$, and $\mathrm{Ge}_{\mathrm{K} \beta}(10.98 \mathrm{keV})$ lines. Since in the PIXE channeling spectra the $\mathrm{Al}_{\mathrm{K} \alpha}$ and $\mathrm{Ga}_{\mathrm{K} \alpha}$ intensities drop down to the noise level, it can be concluded that both $\mathrm{Al}$ and $\mathrm{Ga}$ atoms are fully incorporated into Ge lattice sites, even with a concentration being 4 times higher than the ESS.

More insight into the microstructure is provided by TEM. Please note that the $\mathrm{SiO}_{2}$ capping layer is still present for these samples. Figure 4(a) displays a cross-sectional brightfield TEM image taken from Al-doped Ge after annealing. In this case, both single dislocations within the implanted layer and end-of-range defects are detected [34]. Figure 4(b) shows the $\mathrm{Ge}, \mathrm{Al}$, and $\mathrm{O}$ distributions based on EDXS analysis from a representative surface region, as exemplarily marked by the white square in Fig. 4(a). Aluminum is quite evenly distributed within Ge, showing only few small agglomerates over the implantation depth, which is in good agreement with RBS and PIXE data.

Figures 4(c) and 4(d) show a cross-sectional brightfield TEM micrograph and the corresponding superimposed $\mathrm{Ge}, \mathrm{Ga}$, and $\mathrm{O}$ element distributions obtained from Gahyperdoped Ge. Here, the Ga is completely homogeneously distributed within the implanted layer. Moreover, in the case of the Ga-doped sample, even the end-of-range defects are not detected. For $\mathrm{Al}$ as well as $\mathrm{Ga}$, the recrystallized $\mathrm{Ge}$ is singlecrystalline. This is in contrast to our previous results, where front-side flash lamp annealing was used [15]. Applying

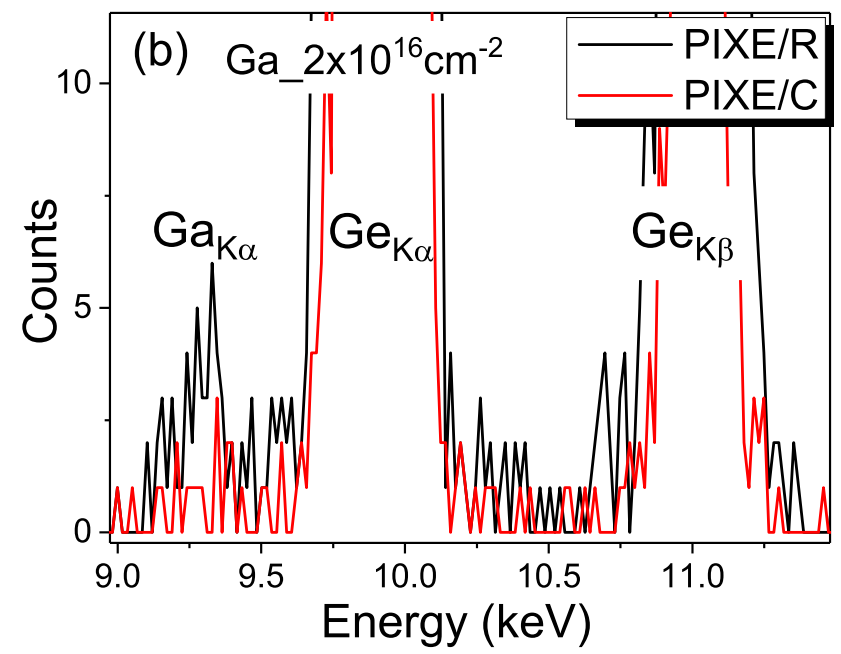

FIG. 3. PIXE spectra of Al- (a) and Ga-hyperdoped Ge (b) followed by rear-side FLA for 20 ms. 

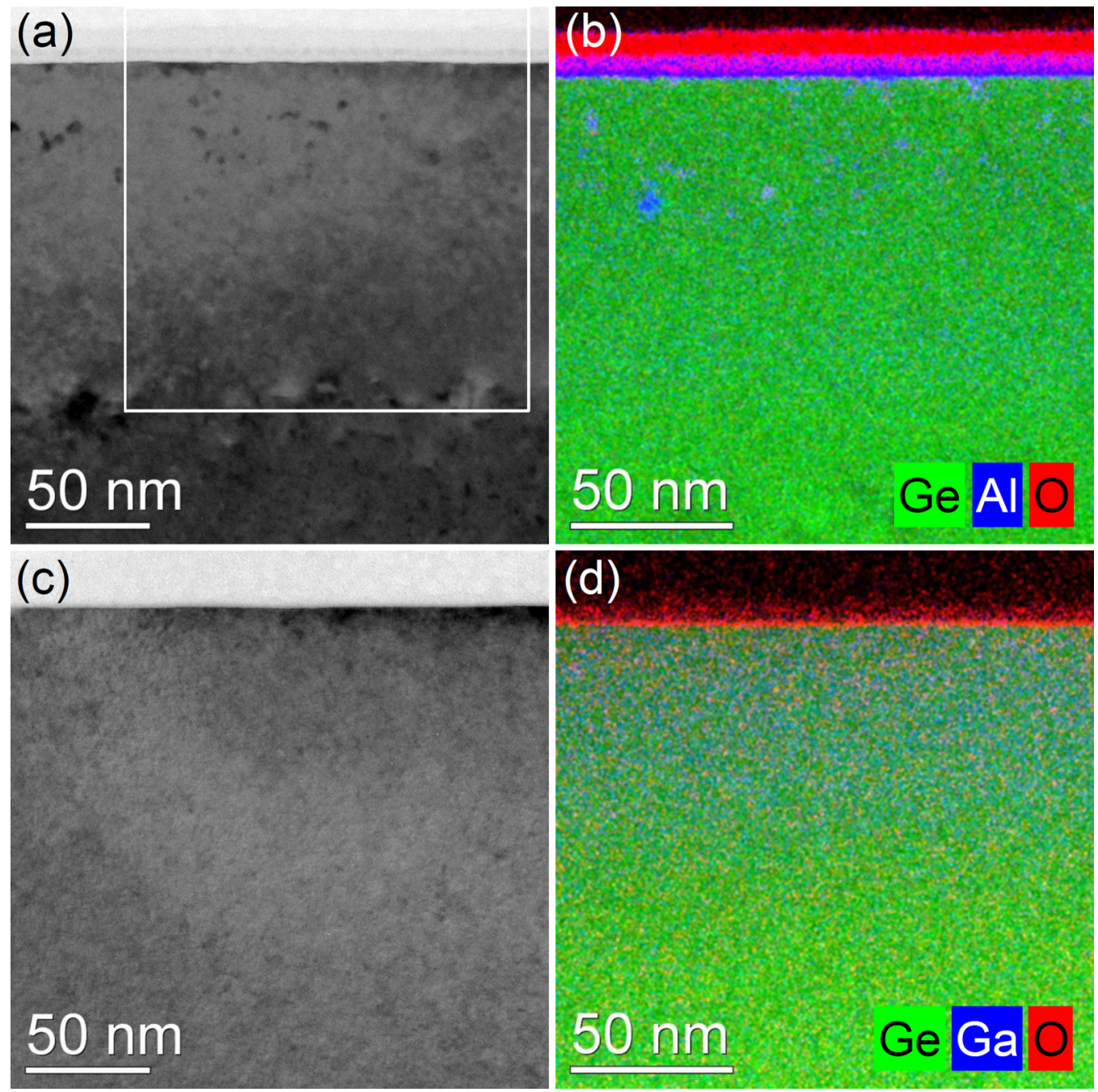

FIG. 4. (a), (c) Cross-sectional bright-field TEM images obtained from Al- and Ga-hyperdoped Ge, respectively, (b), (d) superimposed Ge (green), $\mathrm{O}$ (red), and $\mathrm{Al}$ or $\mathrm{Ga}$ (blue), respectively, element distributions obtained by spectrum imaging analysis based on EDXS in scanning TEM mode for a representative surface region of each sample, as exemplarily marked by the white square in (a).

f-FLA, the implanted layer is composed of polycrystalline $\mathrm{Ge}$ with $\mathrm{Ga}$ clusters and an epitaxial layer which has a thickness of about $70 \%$ of the thickness of the implanted layer. Using r-FLA, we can fully suppress the formation of poly$\mathrm{Ge}$ and $\mathrm{Ga}$ clusters for dopant concnetrations much above the ESS.

\section{B. Superconductivity}

The established electrical parameters of the studied doped layers are summarized in Table I. The carrier concentration was estimated from the Hall-effect measurements. The thickness of the doped layer was determined by RBS measurements. The presented activation efficiency is a ratio between the total acceptor concentration and the carrier concertation estimated from the Hall-effect measurement at $3 \mathrm{~K}$. The presented critical temperatures are taken from Fig. 5. We find that for diluted hyperdoped Ge the minimum carrier concentration needed for superconductivity is in the range of $1 \times 10^{21} \mathrm{~cm}^{-3}$. If we assume that the superconductivity in $p$-type Ge is phonon mediated, for the same doping level the critical temperature should be slightly higher for Al-doped $\mathrm{Ge}$

TABLE I. Summary of the doping level and carrier concentration in hyperdoped Ge obtained for Al- and Ga-doped samples annealed with optimized parameters.

\begin{tabular}{lcccc}
\hline \hline Sample & Dopant concentration & Carrier concentration at $3 \mathrm{~K}$ & Activation efficiency & Critical temperature \\
\hline $\mathrm{Ge}: \mathrm{Al}$ & $\sim 6 \%$ & $10.7 \times 10^{20} \mathrm{~cm}^{-3}$ & $44.5 \%$ & $T_{C} \sim 0.15 \mathrm{~K}$ \\
$\mathrm{Ge}: \mathrm{Ga}$ & $\sim 10 \%$ & $12.6 \times 10^{20} \mathrm{~cm}^{-3}$ & $31.5 \%$ & $T_{C} \sim 0.45 \mathrm{~K}$ \\
\hline \hline
\end{tabular}



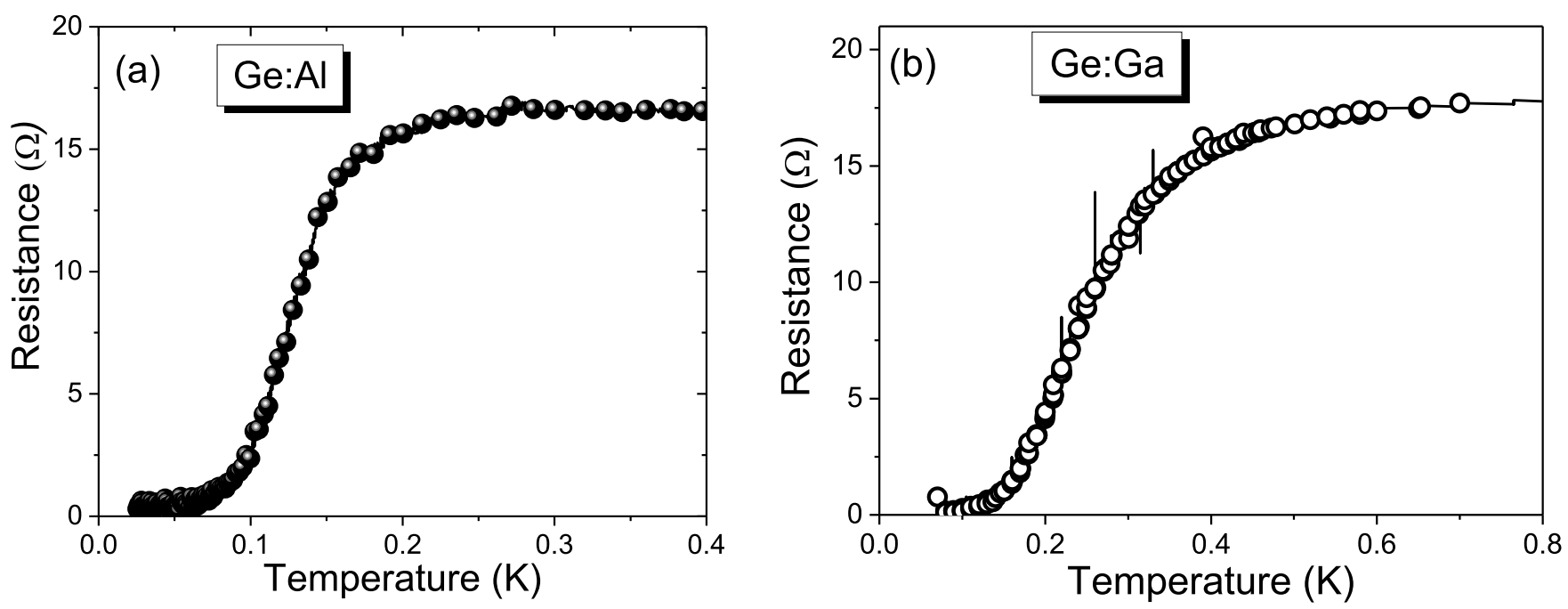

FIG. 5. Temperature dependence of the longitudinal resistance for different samples: (a) low-temperature part of $\mathrm{R}_{x x}$ vs $T$ of superconducting $\mathrm{Ge}$ with $6 \% \mathrm{Al}(\mathrm{a})$ and $\mathrm{Ga}(\mathrm{b})$.

than for the Ga-doped sample due to a stronger phonon coupling. Unfortunately, in hyperdoped Ge it is very challenging to control the carrier concentration keeping the same dopant concentration. In fact, for the doping level above the solid solubility, we are not able to activate $100 \%$ of the implanted element. Therefore experimental verification of theoretical predictions is very challenging. Here we decided to compare samples with similar hole concentrations.

It is worthy to note that we are able to show superconducting Ge hyperdoped with Al. The achieved hole concentration is the highest ever published for Al-doped samples. Figure 5(a) shows the temperature dependence of resistance for Al-hyperdoped Ge. The superconducting temperature is about $150 \mathrm{mK}$. This is much lower than that predicted by calculation ( $T_{\mathrm{C}} \sim 480 \mathrm{mK}$, shown later) but also much below the critical superconducting temperature for $\mathrm{Al}$ thin film or $\mathrm{Al}$ clusters [35]. If we take into account the carrier concentration, which is roughly half of the $\mathrm{Al}$ concentration, the obtained $T_{C}$ is at a reasonable level. The temperature dependence of the resistance for the Ga-doped sample is shown in Fig. 5(b). In Ga-hyperdoped Ge, $T_{C}$ is about $400 \mathrm{mK}$. Presented critical temperatures are obtained from samples annealed from the rear side with a flash energy density of $120 \mathrm{~J} \mathrm{~cm}^{-2}$. The annealing at lower energy densities is not sufficient to recrystallize the implanted layer (no superconductivity), while annealing at higher energy densities activates the dopant diffusion and cluster formation, leading to the superconductivity driven by metallic clusters (see Supplemental Material Figs. 1(b) and 2) [15]. Consequently, the fraction of electrically active dopants, both $\mathrm{Al}$ and $\mathrm{Ga}$, in the substitutional position is much smaller.

\section{Model calculations for the electron-phonon coupling}

According to the BCS theory the critical temperature of a homogeneous superconductor grows with increasing electronphonon coupling strength and Debye temperature. Theoretical calculations demonstrate that in homogeneously doped semiconductors the critical temperature scales with their hole con- centration [36,37]. The critical temperature in diamond can exceed $20 \mathrm{~K}$ for a hole concentration of $10 \%\left(\sim 10^{22} \mathrm{~cm}^{-3}\right)$. Much lower critical temperatures $(<1 \mathrm{~K})$ have been predicted for $\mathrm{Si}$ and $\mathrm{Ge}$. We used the supercell technique to model the hyperdoped Ge. For simulation we have used Ge doped with $\mathrm{Al}$ or $\mathrm{Ga}$ with the concentration of $6.25 \%$, corresponding to the $2 \times 2 \times 2$ supercell with one Ge atom substituted by an $\mathrm{Al}(\mathrm{Ga})$ atom. All calculations were performed within the plane-wave implementation of the local-density approximation (LDA) [38] to density functional theory (DFT) [38-40] in the QUANTUM-ESPRESSO package [41]. Norm-conserving pseudopotentials with a kinetic energy cutoff of 45 Ry were used to represent electron-ion interactions. The $k$-point sampling of the Brillouin zone was set to $6 \times 6 \times 6$ during the structural relaxation and electronic structure calculations, while a dense $12 \times 12 \times 12$ Monkhorst-Pack grid [32] was used for the phonon linewidth calculations. Phonon spectra and electron-phonon coupling constants were calculated using density functional perturbation theory [42] with a $3 \times 3 \times 1$ mesh of $q$ points. For all calculations we have used an optimized lattice constant of Ge supercell of $11.234 \AA$. The hyperdoping of $\mathrm{Ge}$ with $\mathrm{Al}$ or $\mathrm{Ga}$ will lead to a lattice expansion by $0.2 \%$ (11.259 $\AA$ for Al-doped Ge) or lattice compression by $0.1 \%(11.226 \AA$ for $\mathrm{Ga}$ doped $\mathrm{Ge})$, respectively.

Figure 6 shows the electronic structure of Al- and Ga-hyperdoped Ge. According to our calculations for the same dopant concentration, the density of states $N\left(E_{F}\right)$ in Al-hyperdoped Ge $(\sim 2.82$ [states/eV/(supercell)] is slightly higher than in Ga-hyperdoped Ge $(\sim 2.67$ [states/eV/(supercell)]). In both cases the hyperdoped Ge is strongly degenerate, with the Fermi level $\left(E_{F}\right)$ located deep in the valence band $(\sim 0.67 \mathrm{eV}$ below the top of the valenceband maximum). The electronic states near the $E_{F}$ of Al- and Ga-hyperdoped Ge are very similar, and they originate from $p$ states of Ge and acceptor dopants. The hyperdoped $\mathrm{Ge}$ is an sp3 covalent metal.

In a similar way to the electronic structure of hyperdoped $\mathrm{Ge}$, we have calculated the phonon band structure of Aland Ga-hyperdoped Ge (see Fig. 7). By solid blue circles we 


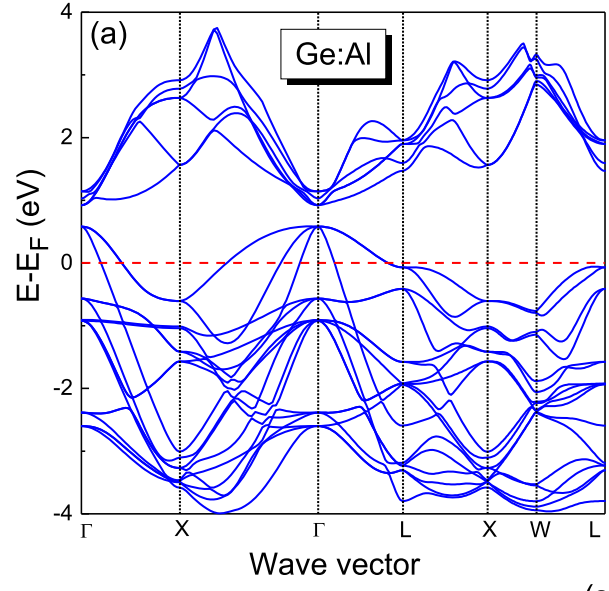

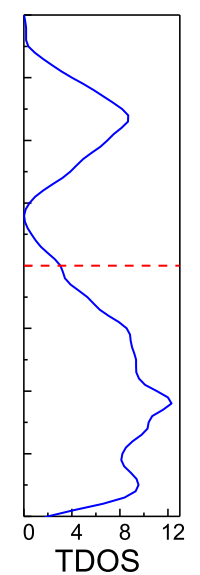

(states/eV/supercell)

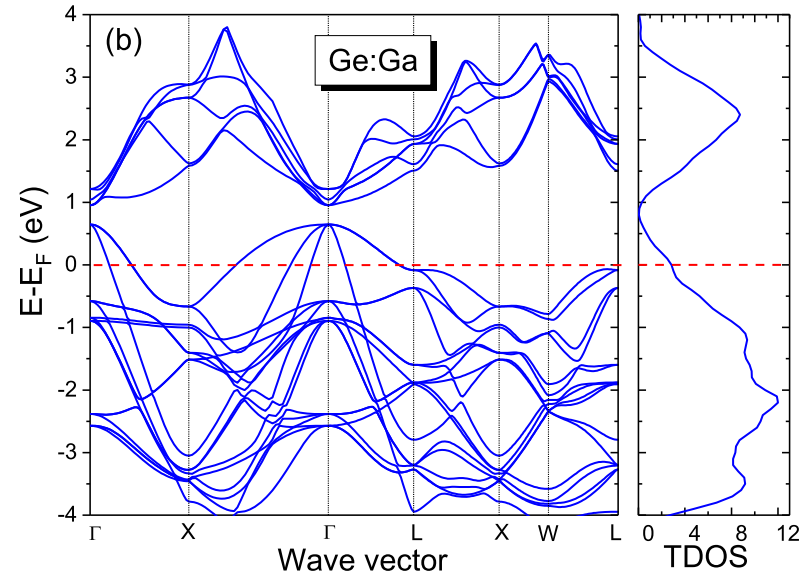

(states/eV/supercell)

FIG. 6. The electronic structure of Al- (a) and Ga-hyperdoped (b) Ge with the corresponding total density of electronic states $N\left(E_{\mathrm{F}}\right)$.

have marked the highest optical phonons at the $\Gamma$ point. In the case of Al-hyperdoped Ge we have found three optical $\mathrm{T}_{2}$ modes located at 256, 267, and $364 \mathrm{~cm}^{-1}$ and one $A_{1}$ optical mode with frequency around $188 \mathrm{~cm}^{-1}$. The strongest electron-phonon coupling strength $\lambda_{\mathrm{vq}}$ at the $\Gamma$ point is at $\mathrm{A}_{1}$ with $\lambda_{v q} \sim 0.16$. The triple degeneracy also produces
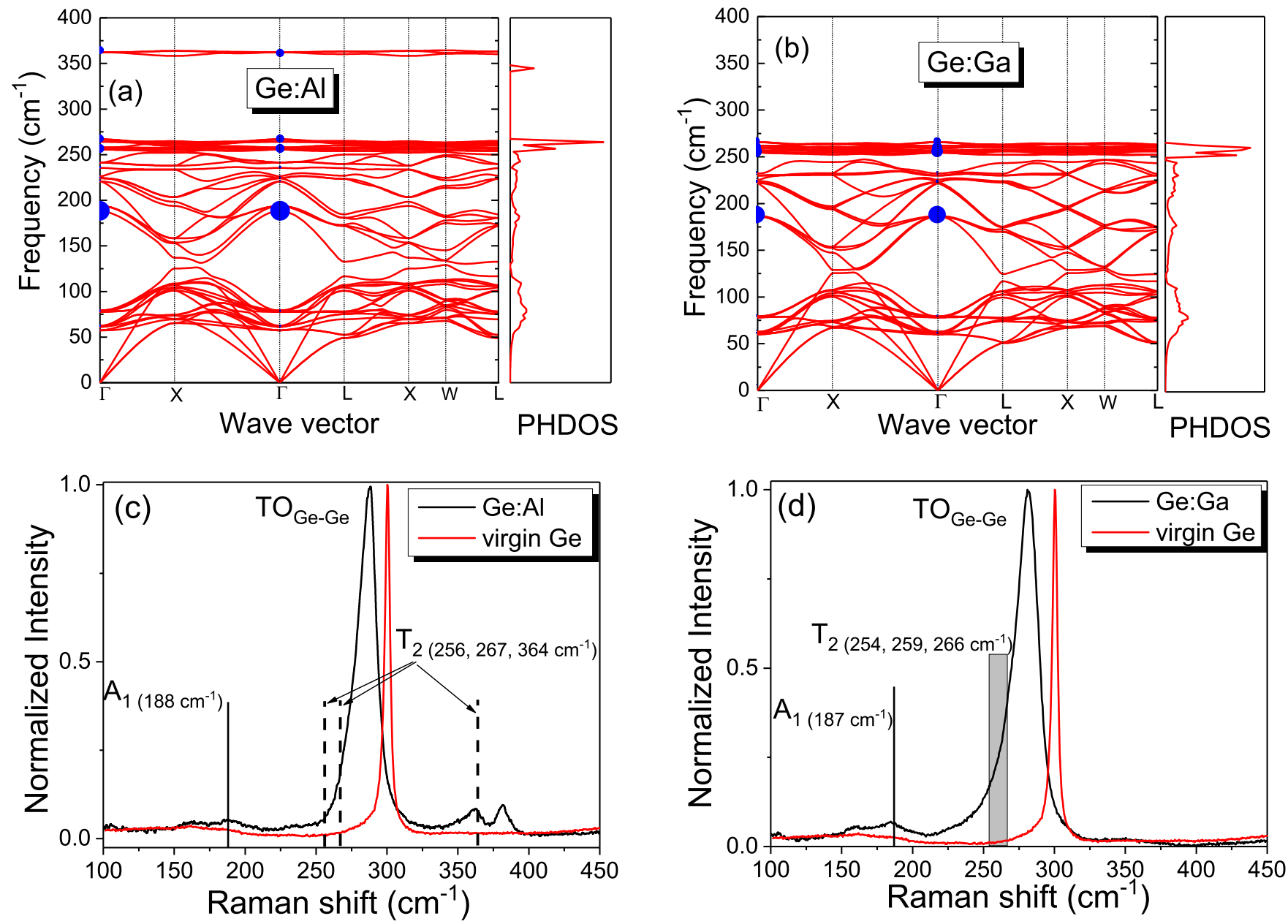

FIG. 7. Phonon band structure with corresponding phonon DOS for Al-hyperdoped Ge (a) and for Ga-hyperdoped Ge (b). The unit of phonon DOS (PHDOS) is states $/\left(\mathrm{cm}^{-1} \times\right.$ supercell). Blue solid circles indicate the partial electron-phonon coupling strength $\lambda_{\mathrm{vq}}$ at the $\Gamma$ point. (c, d) show the Raman spectra obtained from Al- and Ga-hyperdoped Ge, respectively. The Raman spectrum of virgin undoped Ge is shown for comparison. 
electron-phonon coupling strength of about $\lambda_{\mathrm{vq}(\mathrm{q}=\Gamma)} \sim 0.05-$ 0.06 . The phonon structure of Ga-hyperdoped $\mathrm{Ge}$ is very similar to Al-hyperdoped Ge. The $\mathrm{A}_{1}$ optical mode should be located at $187 \mathrm{~cm}^{-1}$, with maximum electron-phonon coupling of about 0.12 . The optical $\mathrm{T}_{2}$ modes in Ga-hyperdoped Ge are located at 254,259 , and $266 \mathrm{~cm}^{-1}$, and the $\lambda_{\mathrm{vq}(\mathrm{q}=\Gamma)}$ is in the range of $0.04-0.07$. The theoretical calculated phonon structure of hyperdoped Ge was verified using micro-Raman spectroscopy. The micro-Raman spectra were collected under 532-nm laser excitation, with a laser power of $3.2 \mathrm{~mW}$ and a focal diameter of about $1 \mu \mathrm{m}$. Figures 7(c) and 7(d) show the Raman spectra obtained from Al- and Ga-hyperdoped Ge, respectively. The transverse-optical phonon mode of Ge-Ge in intrinsic $\mathrm{Ge}$ is located at $300.5 \mathrm{~cm}^{-1}$. After hyperdoping the TO phonon mode of the Ge-Ge vibrational is shifted down to $288.2 \mathrm{~cm}^{-1}$ for the Al-doped sample and down to $281.9 \mathrm{~cm}^{-1}$ for the Ga-doped Ge. This is very close to the theoretically predicted values for the high frequency of the zone-center optical mode in hyperdoped Ge (about $278 \mathrm{~cm}^{-1}$ ). The shift of the TO phonon mode in ultrahigh-doped $\mathrm{Ge}$ and the peak asymmetry is due to the phonon softening and the Fano effect [43-45]. Besides the TO phonon mode we can easily distinguish the $A_{1}$ phonon mode in both samples. The measured peak position of the $A_{1}$ mode located at about $188 \mathrm{~cm}^{-1}$ fits well to the theoretically predicted phonon energy using density of states (DOS) calculation.

Due to the fact that the $\mathrm{T}_{2}$ phonon mode positions are close to the strongest zone-center TO phonon mode it is difficult to distinguish them. But in the case of Al-hyperdoped Ge, the $\mathrm{T}_{2}$ mode at $364 \mathrm{~cm}^{-1}$ is well visible [see Fig. 7(c)]. In Gahyperdoped $\mathrm{Ge}$ all three $\mathrm{T}_{2}$ modes are overlapped with the $\mathrm{TO}$ phonon mode. Next we analyze the electron-phonon coupling. Figures 8(a) and 8(b) show the Eliashberg spectral function and the integrated electron-phonon coupling constant $\lambda(\omega)$. The total $\lambda$ calculated for Al- and Ga-doped Ge are similar and equal to 0.355 and 0.350 , respectively. The calculated logarithmic phonon frequency $\omega_{\log }$ is about $243.6 \mathrm{~K}$ for Aldoped Ge and about 245.1 K for Ga-doped Ge, which is much smaller than the $\omega_{\log }$ in other group IV superconductorsfor example, about $700 \mathrm{~K}$ for $\mathrm{Si}: \mathrm{B}$ and about $1287 \mathrm{~K}$ for boron-doped diamond [45]. Finally, we have calculated the superconducting critical temperature for both samples. We found that the expected $T_{C}$ for Al-doped Ge should be slightly higher than that for the Ga-doped sample, mainly due to slightly higher phonon-carrier coupling. The $T_{C}$ for $\mathrm{Ge}: \mathrm{Al}$ is $0.48 \mathrm{~K}$ and for the Ge:Ga system the $T_{C}$ should be about $0.43 \mathrm{~K}$. According to our calculation(s), the superconductivity in diluted $p$-type hyperdoped Ge should be phonon mediated.

Note that there is significant discrepancy between calculated $T_{C}$ and the experimental values shown in Table I. The theoretical calculation cannot take into account all phenomena which may exist in real samples. In fact, we have a Gaussian distribution of the implanted elements introducing a kind of inhomogeneity into the doped layer which is not accounted for in our calculations and modeling. Next, although the thermal treatment is very short and we were not able to detect big metallic clusters within the implanted Ge layer, we cannot exclude the formation of Ga clusters with a diameter below the resolution limit of our TEM system. Moreover, the ef-
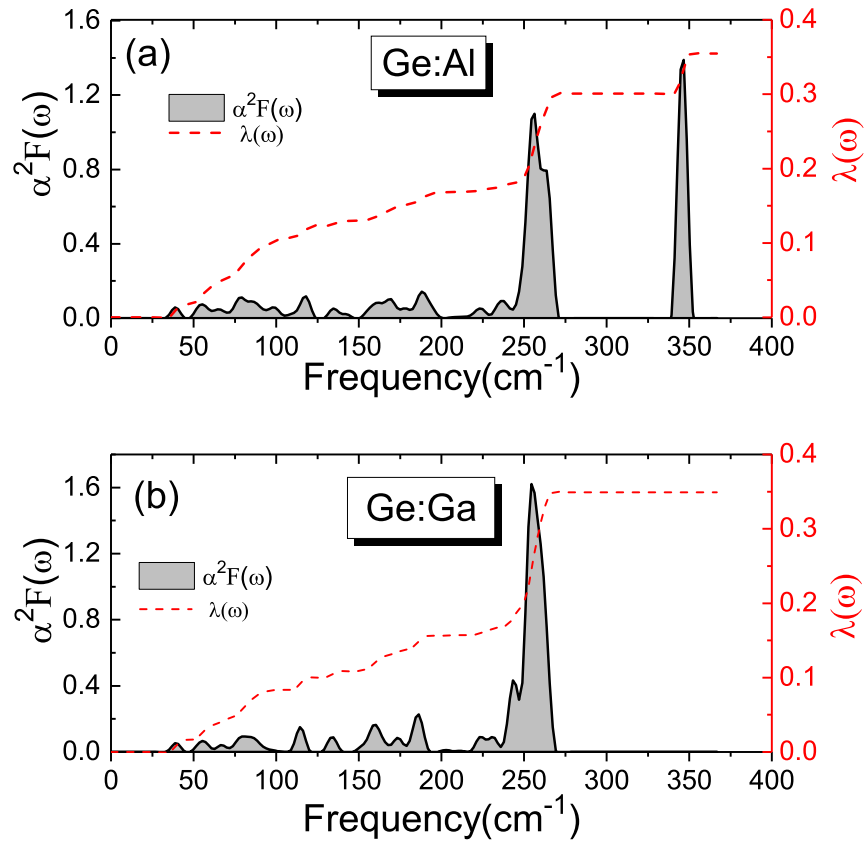

FIG. 8. The total and projected Eliashberg spectral function $\left[\alpha^{2} \mathrm{~F}(\omega)\right]$ for Al-hyperdoped $\mathrm{Ge}$ (a) and for Ga-hyperdoped $\mathrm{Ge}$ (b). The red dashed curves represent the integrated electron-phonon coupling constant $\lambda(\omega)$.

fective carrier concentration is lower than the nominal dopant concentration, which is not taken into account for simulation.

\section{CONCLUSIONS}

We have fabricated single-crystalline Al- and Ga-doped superconducting Ge where the diffusion and clustering of dopants are suppressed by utilization of strongly nonequilibrium thermal processing. Using rear-side FLA, the implanted Ge layers recrystallize epitaxially due to the explosive solidphase epitaxy. The theoretically predicted critical temperatures qualitatively agree with experimental values. With further optimizing dopant concentrations and annealing parameters, our work will pave the way for monolithic integration of superconducting nanocircuits in semiconductor devices.

\section{ACKNOWLEDGMENTS}

Support by the Ion Beam Center (IBC) at HZDR and the funding of TEM Talos by the German Federal Ministry of Education of Research (BMBF) through Grant No. 03SF0451, in the framework of HEMCP, is gratefully acknowledged. We would like to thank Andrea Scholz for XRD measurements and Romy Aniol for TEM specimen preparation. This work was partially supported by the German Academic Exchange Service (DAAD, Project-ID:57216326) and National Science Center, Poland, under Grant No. 2016/23/B/ST7/03451. Partial support by the EU $7^{\text {th }}$ Framework Program, Project No. REGPOT-CT-2013-316014 ("EAgLE") and by the Foundation for Polish Science through the IRA Program cofinanced by the EU within SG OP is also acknowledged. 
[1] E. A. Ekimov, V. A. Sidorov, E. D. Bauer, N. N. Mel'nik, N. J. Curro, J. D. Thompson, and S. M. Stishov, Superconductivity in diamond, Nature (London) 428, 542 (2004).

[2] Y.-P. Shim and Ch. Tahan, Bottom-up superconducting and Josephson junction devices inside a group-IV semiconductor, Nat. Commun. 5, 4225 (2014).

[3] E. Bustarret, C. Marcenat, P. Achatz, J. Kacmarcik, F. Lévy, A. Huxley, L. Ortéga, E. Bourgeois, X. Blasé, D. Débarre et al., Superconductivity in doped cubic silicon, Nature (London) $\mathbf{4 4 4}$, 465 (2006).

[4] T. Herrmannsdörfer, V. Heera, O. Ignatchik, M. Uhlarz, A. Mücklich, M. Posselt, H. Reuther, B. Schmidt, K.-H. Heinig, W. Skorupa et al., Superconducting State in a Gallium-Doped Germanium Layer at Low Temperatures, Phys. Rev. Lett. 102, 217003 (2009).

[5] T. Muranaka, Y. Kikuchi, T. Yoshizawa, N. Shirakawa, and J. Akimitsu, Superconductivity in carrier-doped silicon carbide, Sci. Technol. Adv. Mater., 9, 044204 (2008).

[6] X. Blase, E. Bustarret, C. Chapelier, T. Klein, and C. Marcenat, Superconducting group-IV semiconductors, Nat. Mater. 8, 375 (2009).

[7] K. Iakoubovskii, Recent advances in superconductivity of covalent superconductors, Physica C 469, 675 (2009).

[8] Y. Takano, Superconductivity in CVD diamond films, J. Phys.: Condens. Matter 21, 253201 (2009).

[9] E. Bustarret, Superconductivity in doped semiconductors, Physica C 514, 36 (2015).

[10] D. Cammilleri, F. Fossard, D. Débarre, C. Tran Manh, C. Dubois, E. Bustarret, C. Marcenat, P. Achatz, D. Bouchier, and J. Boulmer, Highly doped $\mathrm{Si}$ and Ge formed by GILD (gas immersion laser doping), From GILD to superconducting silicon, Thin Solid Films 517, 75 (2008).

[11] C. Marcenat, J. Kačmarčík, R. Piquerel, P. Achatz, G. Prudon, C. Dubois, B. Gautier, J. C. Dupuy, E. Bustarret, L. Ortega et al., Low-temperature transition to a superconducting phase in boron-doped silicon films grown on (001)-oriented silicon wafers, Phys. Rev. B 81, 020501(R) (2010).

[12] A. Grockowiak, T. Klein, E. Bustarret, J. Kačmarčík, C. Dubois, G. Prudon, K. Hoummada, D. Mangelinck, T. Kociniewski, D. Débarre et al., Superconducting properties of laser annealed implanted Si:B epilayers, Supercond. Sci. Technol. 26, 045009 (2013).

[13] J. Fiedler, V. Heera, R. Skrotzki, T. Herrmannsdörfer, M. Voelskow, A. Mücklich, S. Oswald, B. Schmidt, W. Skorupa, G. Gobsch et al., Superconducting films fabricated by highfluence $\mathrm{Ga}$ implantation in Si, Phys. Rev. B 83, 214504 (2011).

[14] J. Fiedler, V. Heera, R. Skrotzki, T. Herrmannsdörfer, M. Voelskow, A. Mücklich, S. Facsko, H. Reuther, M. Perego, K.-H. Heinig et al., Superconducting Ga-overdoped Ge layers capped withSiO $\mathrm{S}_{2}$ : Structural and transport investigations, Phys. Rev. B 85, 134530 (2012).

[15] R. Skrotzki, T. Herrmannsdörfer, V. Heera, J. Fiedler, A. Mücklich, M. Helm, and J. Wosnitza, The impact of heavy Ga doping on superconductivity in germanium, Low Temp. Phys. 37, 877 (2011).

[16] K. Hoummada, F. Dahlem, T. Kociniewski, J. Boulmer, C. Dubois, G. Prudon, E. Bustarret, H. Courtois, and D. M.
Débarre, Absence of boron aggregates in superconducting silicon confirmed by atom probe tomography, Appl. Phys. Lett. 101, 182602 (2012).

[17] J. Fiedler, V. Heera, R. Hübner, M. Voelskow, S. Germer, B. Schmidt, and W. Skorupa, High-fluence Ga-implanted siliconThe effect of annealing and cover layers, J. Appl. Phys. 116, 024502 (2014).

[18] F. Dahlem, P. Achatz, O. A. Williams, D. Araujo, E. Bustarret, and H. Courtois, Spatially correlated microstructure and superconductivity in polycrystalline boron-doped diamond, Phys. Rev. B 82, 033306 (2010).

[19] G. Zhang, T. Samuely, J. Kačmarčík, E. A. Ekimov, J. Li, J. Vanacken, P. Szabó, J. Huang, P. J. Pereira, D. Cerbu et al., Bosonic Anomalies in Boron-Doped Polycrystalline Diamond, Phys. Rev. Appl. 6, 064011 (2016).

[20] V. F. Gantmakher and V. T. Dolgopolov, Superconductorinsulator quantum phase transition, Phys. Usp. 53, 1 (2010).

[21] V. Heera, J. Fiedler, M. Voelskow, A. Mücklich, R. Skrotzki, T. Herrmannsdörfer, and W. Skorupa, Superconductor-insulator transition controlled by annealing in $\mathrm{Ga}$ implanted $\mathrm{Si}$, Appl. Phys. Lett. 100, 262602 (2012).

[22] V. Heera, J. Fiedler, R. Hübner, B. Schmidt, M. Voelskow, W. Skorupa, R. Skrotzki, T. Herrmannsdörfer, J. Wosnitza, and M. Helm, Silicon films with gallium-rich nanoinclusions: From superconductor to insulator, New J. Phys. 15, 083022 (2013).

[23] V. Heera, J. Fiedler, and W. Skorupa, Large magnetoresistance of insulating silicon films with superconducting nanoprecipitates, AIP Adv. 6, 105203 (2016).

[24] G. Impellizzeri, S. Mirabella, A. Irrera, M. G. Grimaldi, and E. Napolitani, Ga-implantation in Ge: Electrical activation and clustering, J. Appl. Phys. 106, 013518 (2009).

[25] V. Heera, A. Mücklich, M. Posselt, M. Voelskow, C. Wündisch, B. Schmidt, R. Skrotzki, K. H. Heinig, T. Herrmannsdörfer, and W. Skorupa, Heavily Ga-doped germanium layers produced by ion implantation and flash lamp annealing: Structure and electrical activation, J. Appl. Phys. 107, 053508 (2010).

[26] L. Rebohle, S. Prucnal, and W. Skorupa, A review of thermal processing in the subsecond range: Semiconductors and beyond, Semicond. Sci. Technol. 31, 103001 (2016).

[27] S. Prucnal, L. Rebohle, and W. Skorupa, Doping by flash lamp annealing, Mater. Sci. Semicond. Process., 62, 115-127 (2017).

[28] S. Prucnal, F. Liu, M. Voelskow, L. Vines, L. Rebohle, D. Lang, Y. Berencén, S. Andric, R. Boettger, M. Helm et al., Ultra-doped n-type germanium thin films for sensing in the mid-infrared, Sci. Rep. 6, 27643 (2016)

[29] V. Heera, J. Fiedler, M. Naumann, R. Skrotzki, S Kölling, L. Wilde, T. Herrmannsdörfer, W. Skorupa, J. Wosnitza, and M. Helm, Depth-resolved transport measurements and atom-probe tomography of heterogeneous, superconducting Ge:Ga films, Supercond. Sci. Technol. 27, 055025 (2014).

[30] G. Impellizzeri, E. Napolitani, S. Boninelli, V. Privitera, T. Clarysse, W. Vandervorst, and F. Priolo, Aluminium implantation in germanium: Uphill diffusion, electrical activation, and trapping, Appl. Phys. Express 5, 021301 (2012). 
[31] J. F. Ziegler, J. P. Biersack, and U. Littmark, The Stopping and Range of Ions in Matter (Pergamon Press, New York, 1985).

[32] See Supplemental Material at http://link.aps.org/supplemental/ 10.1103/PhysRevMaterials.3.054802 for more details about samples fabrication and an influence of the dopant concentration on the superconducting states in Ge.

[33] M. Posselt and A. Gabriel, Atomistic simulation of amorphous germanium and its solid phase epitaxial recrystallization, Phys. Rev. B 80, 045202 (2009).

[34] A. Claverie, S. Koffel, N. Cherkashin, G. Benassayag, and P. Scheiblin, Amorphization, recrystallization and end of range defects in germanium, Thin Solid Films 518, 2307 (2010).

[35] A. Bezryadin and B. Pannetier, Nucleation of superconductivity in a thin film with a lattice of circular holes, J. Low Temp. Phys. 98, 251 (1995).

[36] L. Boeri, J. Kortus, and O. K. Andersen, Three-Dimensional MgB2-Type Superconductivity in Hole-Doped Diamond, Phys. Rev. Lett. 93, 237002 (2004).

[37] L. Boeri, J. Kortus, and O. K. Andersen, Electron-phonon superconductivity in hole doped diamond: A first principles study, J. Phys. Chem. Solids 67, 552 (2006).
[38] D. M. Ceperley and B. J. Alder, Ground State of the Electron Gas by a Stochastic Method, Phys. Rev. Lett. 45, 566 (1980).

[39] P. Hohenberg and W. Kohn, Inhomogeneous electron gas, Phys. Rev. 136, B864 (1964).

[40] W. Kohn and L. J. Sham, Self-consistent equations including exchange and correlation effects, Phys. Rev. 140, A1133 (1965).

[41] P. Giannozzi et al., QUANTUM EXPRESSO: A modular and opensource software project for quantum simulations of materials, J. Phys.: Condens. Matter 21, 395502 (2009).

[42] S. Baroni, S. de Gironcoli, A. Dal Corso, and P. Giannozzi, Phonons and related crystal properties from density-functional perturbation theory, Rev. Mod. Phys. 73, 515 (2001).

[43] X. Blase, C. Adessi, and D. Connetable, Role of the Dopant in the Superconductivity of Diamond, Phys. Rev. Lett. 93, 237004 (2004).

[44] H. J. Xiang, Zhenyu Li, Jinlong Yang, J. G. Hou, and Qingshi $\mathrm{Zhu}$, Electron-phonon coupling in a boron-doped diamond superconductor, Phys. Rev. B 70, 212504 (2004).

[45] J. Dai, Z.-Y. Li, and J.-L. Yang, Electron-phonon coupling in gallium-doped germanium, Chin. Phys. Lett. 27, 086102 (2010). 